\title{
ASSESSMENT OF OSSTELL AND PERIOTEST SYSTEMS IN MEASURING IMMEDIATE DENTAL IMPLANTS STABILITY
}

\author{
Mohamed Karem El-Sawy*, Mahmoud Taha El-Destawy**, Usama Mohmmed Madany ${ }^{* * *}$
}

\begin{abstract}
Objective: Objective measurement of implant stability through various techniques could assist the clinician in an optimal treatment plan, support decisions regarding implant loading, provides better case documentation, support good communication and increase trust. Therefore, it is very important to detect the accuracy of the most popular noninvasive devices (Periotest, Osstell) that are used in measuring implant stability for evaluating the long-term success of implant. The aim of this study was to evaluate the Osstell and Periotest devices' accuracy in measuring the immediate dental implants stability. Subjects \& Methods: The stability was measured for 32 immediate implants (16 males, 16 females with age range from 25 to 45 years old) by the two devices at the time of implant placement, 6 and 12 months after surgery for each observer. Measurements were performed on four groups according to the site of placement of the immediate implants and classified as anterior area of the maxilla (group A), posterior area of the maxilla (group B), anterior area of the mandible (group C) and posterior area of the mandible (group D). Results: Intra class Correlation Coefficient indicated less reproducibility by the Periotest device compared to Osstell device. This result was confirmed by using the Quantile- Quantile Plot and the distribution of Osstell values was almost linear to the normal distribution in the $1^{\text {st }}$ and $2^{\text {nd }}$ measurements. The Periotest values differed from the normal distribution. Conclusion: Both the Osstell (ISQs) and Periotest (PTVs) systems may indicate reliable assessments of implant stability. The Osstell system may exhibit better reliability compared to Periotest in measuring the implant stability at time of placement and during healing, up to 12 months.
\end{abstract}

Key Words: Ostell, Periotest, Immediate implant stability

\section{INTRODUCTION}

Osseointegration is defined as a direct bone anchorage to an implant body which can provide a foundation to support prosthesis and is also a measure of implant stability which occurs in two stages; primary and secondary. Implant stability is a requisite characteristic of osseointegration without it, long term success cannot be achieved. Continuous monitoring in a quantitative and objective manner is important to determine the status of implant stability ${ }^{(1,2)}$.

Primary stability is a mechanical fact, defined as the absence of clinical mobility at the time of insertion, and has an important role on immediate loading success ${ }^{(3)}$. It is important for successful bone integration of dental implants. On the other hand, Secondary stability offers biological stability through bone regeneration and remodeling and is affected by primary stability ${ }^{(4,5)}$. It is important to check the implant stability before going for immediate loading.

Although various diagnosis analysis of implants has been employed and several research and development projects have been already made in this field, measuring implant stability remains a challenge in dentistry. In modern implantology,

\footnotetext{
* BDS, 2010 G, Faculty of Oral and Dental Medicine, MUST University, Dentist: Ministry of Health.

** Lecturer, Department of Oral Medicine, Periodontology, Diagnosis and Oral Radiology. Faculty of Dental Medicine, (Boys - Cairo), Al-Azhar University.

*** Professor, Department of Oral Medicine, Periodontology, Diagnosis and Oral Radiology. Faculty of Dental Medicine, (Boys - Cairo), Al-Azhar University.
} 
with the introduction of surgical and prosthetic protocols such as the immediate placement of implant in fresh post-extraction sockets and immediate functional loading, it is very important to quantify implant stability at various timeframes in order to have a long-term prognosis for implants placed.

Objective measurement of implant stability through various techniques could assist the clinician in an optimal treatment plan, support decisions regarding implant loading protocols on a patient to patient basis, provides better case documentation, support good communication and increase $\operatorname{trust}^{\left({ }^{(}\right)}$. Various methods developed to assess implant stability are classified to invasive and noninvasive. Invasive methods are limited to nonclinical studies because of ethical concerns include histological analysis (evaluation of bone-implant contact in specimen), tensional test, removal torque analysis, Reverse Torque Test (RTT), and push out, pull out test. Noninvasive methods are the surgeon's perception, radiographic analysis (imaging techniques), Cutting Torque Resistance Analysis (CRA) for primary stability, insertion torque, seating torque, modal analysis, percussion test, Pulsed Oscillation Waveform (POWF), Periotest, Resonance Frequency Analysis (RFA), and magnetic technology ${ }^{(7)}$.

\section{SUBJECTS AND METHODS}

The stability was measured for each implant by the two devices at the time of implant placement, 6 and 12 months after surgery. Measurements were taken for 16 immediate implants in male and 16 immediate implants in female with age range from 25 to 45 years old Measurements were performed on four groups according to the site of placement of the immediate implants and classified as anterior area of the maxilla (group A), posterior area of the maxilla (group B), anterior area of the mandible (group C) and posterior area of the mandible (group D). In each group 8 immediate implants were placed separately.
All the implants used in this study were root form threaded J dental implants and implant insertion was performed according to manufacturer's instruction. All smart pegs used for Osstell measurements were produced by Osstell designed for J dental implant.

\section{The Osstell measurement:}

Measurements (ISQs) were taken by Osstell device produced by Osstell AB, Goteborg, Sweden. The resonance frequency value of the implant fixture was measured through the transducer (smart peg) with maintain a distance of approximately 1-3 $\mathrm{mm}$, angle of 90 degrees, and $3 \mathrm{~mm}$ above the soft tissue which is mounted directly to the fixture with a screw and each implant had a new smart peg. The measurements were performed in the mesial, distal, buccal and palatal/lingual directions, for each inserted implant. These measurements were taken twice by two different observers, Observer 1 and Observer 2. The mean of the measurements in all directions was regarded as the representative ISQ of the implant for each observer accordantly. Follow-up was scheduled throughout the study period. All implants were functionally loaded at 6 months during the follow up period and the 12 months measurements were taken after removal of prosthesis (screw retained abutment) and using the transducer (smart peg).

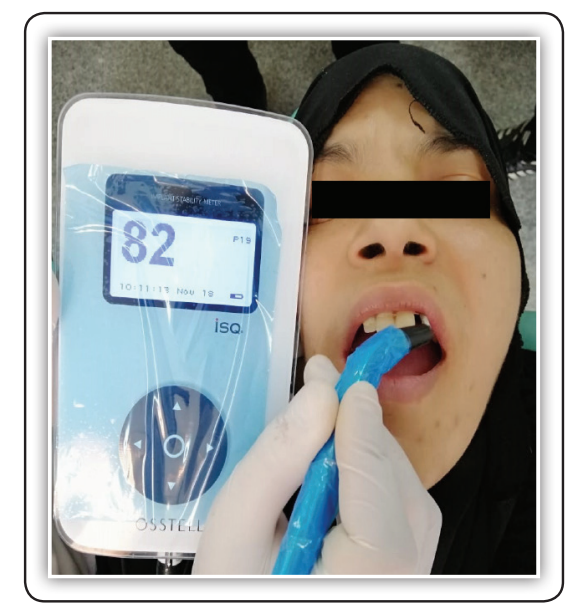

FIG (1) Implant stability measurement with Osstell device 


\section{The Periotest measurement:}

Measurements (PTVs) were taken by Periotest device from Semens AG, Benshein, Germany, (figure 1) Hand piece was adjusted with perpendicular contact angle not exceeding 20 degree on the abutment and maintained the distance between the rod and the abutment at $0.6-2.0 \mathrm{~mm}$, according the published guidelines. All measurements were performed in the mesial, distal, buccal and palatal/ lingual directions, for each inserted dental implant. These measurements were taken twice by the two observers, Observer 1and Observer 2. The mean of measurements in all directions was regarded as the PTV of the implant for each observer accordantly. Follow-up was scheduled during the study period. The measurements at time of placement and at 6 months were taken on the J Dental implant abutment and at 12 months were taken without removal of prosthesis.

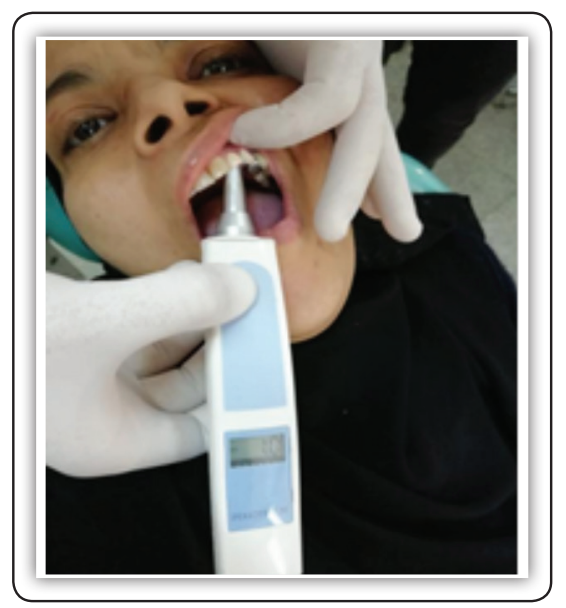

FIG (2) Implant stability measurement with Periotest device upon the final prothesis

\section{RESULTS}

At time of implant placement Intra class Correlation coefficient (ICC) was 0.967 for Osstell and 0.897 for Periotest. After 6 months the ICC was 0.998 and 0.935 for Osstell and Periotest respectively, while, after 12 months the ICCs were 0.998 and 0.837 . The ICC data indicated superiority of Osstell compared to Periotest in the anterior area of maxilla, (Table 1).

TABLE (1): Intra class Correlation coefficient for different parameters regarding the two observers in anterior maxilla.

\begin{tabular}{|c|c|c|c|c|}
\hline \multirow{3}{*}{ 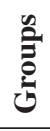 } & \multirow{3}{*}{ Time } & \multicolumn{3}{|c|}{ Observer 1 vs. Observer 2} \\
\hline & & \multirow{2}{*}{ ICC } & \multicolumn{2}{|c|}{$95 \% \mathrm{CI}$} \\
\hline & & & $\mathbf{L L}$ & $\mathbf{U L}$ \\
\hline \multirow{3}{*}{$\begin{array}{l}\overline{\bar{v}} \\
\text { 苟 } \\
0 \\
0\end{array}$} & At implant placement & 0.967 & 0.847 & 0.993 \\
\hline & $6^{\text {th }}$ month & 0.998 & 0.992 & 1.000 \\
\hline & $12^{\text {th }}$ month (At loading) & 0.998 & 0.989 & 1.000 \\
\hline \multirow{3}{*}{$\begin{array}{l}\overrightarrow{0} \\
\stackrel{0}{0} \\
\stackrel{0}{0} \\
0\end{array}$} & At implant placement & 0.897 & 0.574 & 0.979 \\
\hline & $6^{\text {th }}$ month & 0.935 & 0.711 & 0.987 \\
\hline & $12^{\text {th }}$ month (At loading) & 0.837 & 0.386 & 0.965 \\
\hline
\end{tabular}

ICC: Intra class Correlation coefficient

Less than 0.75 poor

0.75 to less than 0.90 moderate agreement

0.90 or greater high agreement

Intra class Correlation Coefficient (ICC) at implant placement was 0.986 for Osstell and 0.921 for Periotest. After 6 months was 0.956 and 0.861 respectively, while, after 12 months was 0.927 and 0.805 . The ICC at implant placement, at 6 months and at 12 months announces predomination of Osstell device measurements in posterior area of maxilla, (Table 2).

TABLE (2) : Intra class Correlation Coefficient for different parameters regarding the two observers in posterior maxilla.

\begin{tabular}{|c|c|c|c|c|}
\hline \multirow{3}{*}{ 苛 } & \multirow{3}{*}{ Time } & \multicolumn{3}{|c|}{ Observer 1 vs. Observer 2} \\
\hline & & \multirow{2}{*}{ ICC } & \multicolumn{2}{|c|}{$95 \% \mathrm{CI}$} \\
\hline & & & $\mathbf{L L}$ & UL \\
\hline \multirow{3}{*}{$\begin{array}{l}\bar{\nabla} \\
\stackrel{0}{0} \\
0 \\
0\end{array}$} & At implant placement & 0.986 & 0.933 & 0.997 \\
\hline & $6^{\text {th }}$ month & 0.956 & 0.798 & 0.991 \\
\hline & $12^{\text {th }}$ month (At loading) & 0.927 & 0.682 & 0.985 \\
\hline \multirow{3}{*}{ 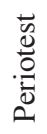 } & At implant placement & 0.921 & 0.659 & 0.659 \\
\hline & $6^{\text {th }}$ month & 0.861 & 0.456 & 0.971 \\
\hline & $12^{\text {th }}$ month (At loading) & 0.805 & 0.299 & 0.958 \\
\hline
\end{tabular}

ICC: Intra class Correlation coefficient

Less than 0.75 poor

0.75 to less than 0.90 moderate agreement

0.90 or greater high agreement 
The ICCs data indicated more accuracy of Osstell measurements than Periotest measurements throughout the study in posterior area of mandible. The Q-Q plots showed that the distribution of the Osstell values by the two observers was more linear than the distribution of Periotest values. This indicated more accuracy of Osstell device measurement's distribution by the first and second observers than Periotest device measurement's distribution.

\section{DISCUSSION}

Many studies have indicated the presence of a correlation between PTVs and ISQs, and it has been reported that both methods are useful in the evaluation of implant stability along with other clinical tests. It has been proposed that both methods should be combined to detect which more accurate in detecting the stability of implants ${ }^{(8)}$. The laboratory experiments with the Osstell and the Periotest devices showed a statistically linear association between measurements, with high statistical correlation coefficients of -0.9 and $-0.8^{(9)}$. The correlation of both methods in clinical trial is clearly less pronounced. In clinical use, the examiner is limited by access, space and patient compliance, unlike in laboratory experiment, where a standardized measuring set-up permits constant conditions. Aparicio et al. ${ }^{(10)}$ presented that the validity and relevance of both Osstell and Periotest for clinical use must be questioned.

In diagnostic and clinical decision making, a key factor is A diagnostic procedure controlling the choice of the therapeutic method employed needs to be predictive and reliable. The present study was designed to detect the accuracy of both devices in repeated measurements clinically to know the extent to which a single measuring procedure yields the same results during a repeated trial.

The study was performed on immediate implants placed in the maxilla and the mandible to evaluate the measurements difference according to bone quality.
Moreover, there were 4 groups; anterior (group A) and posterior (group B) in maxilla, and anterior (group C) and posterior (group D) in mandible. The measurements were recorded using Osstell and Periotest at time of implant placement, at 6th month and at 12th month. Each implant was evaluated with each device by two different evaluators.

In general, the measurements in the present study revealed that mandible had lower PTVs and higher ISQs values than the maxilla. This observation agrees with Ji-Su oh et al. ${ }^{(11)}$ who found the same results. These data might be attributed to the different bone density between the 2 jaws, and the result corresponded to the observation that PTV and ISQ are affected by bone quality, bone density and the degree of compact bone which are higher in the lower than the upper jaw ${ }^{(12)}$.

The ICC in this study for Osstell's measurement in anterior and posterior maxilla showed highly reliability at time of insertion than at 6 months and 12 months which is in agreement with Denis et al. ${ }^{(13)}$ who analyzed the implant stability quotient (ISQ) results of the resonance frequency (RF) during the first day of surgery and 3 months after dental implant placement in the maxilla. The results demonstrate that the influence and reliability of measured Osstell ISQ values at that time of period were reliable and predictable for the oral implant outcomes.

In this study, regarding measuring the stability separately for anterior and posterior region of the jaws, considering the intraoral environment and the pen-grip shaped handpiece of the Periotest, it was clear that, it can be used quite easily for the anterior region. However, its use for the posterior region is extremely difficult because of the presence of buccal mucosa.$^{(14)}$ Moreover, the ICC results encountered in the present study announced the superiority of Periotest at all study periods in measuring implant stability in anterior region than posterior region. This observation agrees with Salonen et al. ${ }^{(15)}$ who reported that PTVs in anterior region of maxilla was higher than posterior region. These results are 
parallel to those of Derhami et al. ${ }^{(16)}$ who reported the same limitation and Periotest device is effective evaluation method once difficulty of controlling impact was solved.

In the present study the ICC for Periotest measurement at time of insertion showed better reliability than ICC of the measurements at 6 months 12 months, which might be due to bone loss during that time. This may coincide with results of several previous in vivo and in vitro studies. Carnin et al. ${ }^{(17)}$ who examined the Periotest as a possible replacement for outdated, inconsistent dental implant stability diagnosis tools. Periotest failed to diagnose implants with progressive bone loss in patients, because its values remain the same until the bone loss is virtually terminal. Therefore, they concluded that the information gained from application of the Periotest would have to be combined with other clinically harvested information in order to determine the true status of an implant. Lachmann et al. ${ }^{(18)}$ compared Osstell and Periotest by in vitro study and demonstrated that both methods are useful in the evaluation of implant stability in different bone densities but the Osstell was more precise than the Periotest to determine the actual dental implant stability at peri-implant bone defects. The same result also was obtained by Winter et al. ${ }^{(19)}$ who investigated the correlation between the two devices through the finite element study and demonstrated that Periotest values had only good correlation with implant stability in cases when there is no bone loss.

In contrast, Khalaila et al. ${ }^{(20)}$ performed a prospective clinical study to investigate implant stability by using PTVs, as well as changes in stability and peri-implant marginal bone levels (as measured by radiographs) over a 3-year follow-up period. The exploratory hypothesis was that there is a significant correlation between PTVs and bone loss around the implant and PTVs can provide predictive information about marginal bone-level changes and implant stability over time. The results denoted that the Periotest was a reliable device for assessing implant stability and providing predictive information about marginal bone level changes around an implant.

In the present study, the overall evaluation of the two devices in measuring the immediate dental implants stability using the Intra class Correlation Coefficient indicated less reproducibility by the Periotest compared to Osstell. This result was confirmed by using the Quantile- Quantile Plot, which visualizes whether a data sample follows a normal distribution. The distribution of Osstell values was almost linear to the normal distribution in the 1st and 2nd measurements. The Periotest values differed from the normal distribution. The differences were larger with a wider distribution of repeated data and further far from the curve. This indicated the possibility of that Osstell may have more accuracy in measuring dental implants stability than Periotest. This is in accordance with our results of Zix et al. ${ }^{(21)}$ who concluded that the Osstell instrument seemed to be more precise than the Periotest.

The current results are in accordance with those of Al-Jetaily et al. ${ }^{(22)}$ who investigated the sensitivity and reliability of the Osstell system compared to the Periotest system in implant bone simulated conditions (vitro study). The encountered results also agree with Gul Buyukguclu et al. (23) who reported highly reliable of the Osstell system.

In contrast to presented results, Bilhan et al. ${ }^{(24)}$ who reported that there was no significance when Periotest was compared with ISQ values observed for the same implants. Moreover, Periotest could show a high degree for measurements, especially, conducted and applied at buccal directions. Also, Won-Suk et al. ${ }^{25)}$ in another study which is in contrast with the present results, investigated the reproducibility of Periotest values (PTVs) and implant stability quotients (ISQs) in-vitro. They found that, although both the PTVs and ISQs might indicate reliable assessments of implant stability the 
PTVs were found to be more consistent than ISQs among the evaluators. However, these studies were performed in vitro where application of Periotest is much more convenient than in vivo.

In clinical studies, numerous variables must be controlled in gathering clinical data. Both the reproducibility of instruments used to collect data and the way the instruments are used by clinicians can influence the results. This problem is even more significant when different dentists with different levels of clinical experience utilize a different instrument from the same manufacturer to evaluate the implant stability ${ }^{(25)}$.

Within the limits of the current study, the ISQs were found to be more consistent than PTVs among the evaluators. However, both the Osstell PTVs and Periotest systems ISQs may indicate reliable assessments of implant stability because of small differences in the means among evaluators and narrow distributions of repeated data within each evaluator. The Osstell instrument seems to be more precise and reliable compared to Periotest in detecting the changes in the fixture interface stiffness during healing.

\section{REFERENCES}

1. Albrektsson T, Zarb G, Worthington P, Eriksson AR. The long-term efficacy of currently used dental implants:Areview and proposed criteria of success. Int J Oral Maxillofac Implants 1986; 1:11-25.

2. Meredith N. Assessment of implant stability as a prognostic determinant. Int J Prosthodont 1998; 11: 491-501.

3. Brunski JB. Biomechanical factors affecting the bonedental implant interface. Clin Mater 1992; 10:153-201.

4. Sennerby L, Roos J. Surgical determinants of clinical success of osseointegrated oral implants: A review of the literature. Int J Prosthodont 1998; 11:408-20.

5. Cochran DL, Schenk RK, Lussi A, Higginbottom FL, Buser D. Bone response to unloaded and loaded titanium implants with a sandblasted and acid-etched surface: A histometric study in the canine mandible. J Biomed Mater Res 1998; 40:1-11.
6. Mall N, Dhanasekar B, Aparna IN. Validation of implant stability: A measure of implant permanence. Indian J Dent Res 2011;22:462-7.

7. Swami V, Vijayaraghavan V, Swami V. Current trend to measure implants stability. J Indian Prosthodont Soc 2016; $16: 124-30$

8. Azzaldeen Abdulgani ,Mai Abdulgani ,Abu-Hussein Muhamad; Immediate Restoration of Single Implants Replacing Lateral Incisor Compromised by Int Res J of Dent Med Sciences.2017; 16,5:131-37.

9. Agliardi E, Panigatti S, Clerico M, Villa C, Malo P. Immediate rehabilitation of the edentulous jaws with full fixed prostheses supported by four implants: Interinm results of a single cohort prospective study. Clinic O.Implants Res. $2010 ; 21: 459-65$.

10. Aparicio, C., Lang, N. P. \& Rangert, B. Validity and clinical significance of biomechanical testing of implant/bone interface. Clin Oral Impl Res. 2006; 17 Suppl 2: 2-7.

11. Oh, J.S., Kim, S.G., Lim, S.C. \& Ong, J. L. A comparative study of two noninvasive techniques to evaluate implant stability:Periotest and Osstell mentor. O.Surg, O.Med, O.Path, O. Radio, and Endo. 2009; 107: 513-18.

12. Branemark, R., Ohrnell, L. O., Skalak, R., Carlsson, L. \& Bronemark, P. I. Biomechanical characterization of osseointegration: An experimental in vivo investigation in the beagle dog. J of Orthopedic res. 1998; 16.

13. Denis baftijari, Alberto Benedetti, Antonio Kirov et al. assessment of primary and secondary implant stability by resonance frequency analysis in anterior and posterior segments of maxillary edentulous ridges. J of imab. 2018; 2-24.

14. Truhlar, R.S., Morris, H.F. \& Ochi, S. Stability of the boneimplant complex. Results of longitudinal testing to 60 months with the Periotest device on endosseous dental implants. Annals/Journal of Periodontology 2000; 5: 42-55.

15. Salonen, M.A., Raustia, A.M., Kainulainen, V. \& Oikarinen, K.S. Factors related to Periotest values in endosseal implants: a 9-year follow-up. Journal of Clinical Periodontology 1997; 24: 272-77.

16. Derhami, K., Wolfaardt, J.F., Faulkner, G. \& Grace, M. Assessment of the Periotest device in baseline mobility measurements of craniofacial implants. International Journal of Oral \& Maxillofacial Implants 1995; 10: 221-29.

17. A. Norman Cranin et al. Evaluation of the Periotest as a diagnostic tool for dental implants. J of Oral Imp. Vol. XXIV No. 3.1998; 139-42. 
18. Lachmann, S., Laval, J. Y., Jager, B., Axmann, D., GomezRoman, G., Groten, M. \& Weber, H. Resonance frequency analysis and damping capacity assessment. Part 2: Periimplant bone loss follow-up. An in vitro study with the Periotest and osstell instruments. Clin Oral Implants Res. 2006; 17: 80-84.

19. Winter W., Mohrle S., Holst S., \& Karl, M. Parameters of implant stability measurements based on resonance frequency and damping capacity: A comparative finite element analysis. The Int J Oral Maxillofac Implants. 2010; 25: 532-39.

20. Waseem Khalaila, Minaem Nasser, Zeev Ormianer. Evaluation of the relationship between Periotest values, marginal bone loss, and stability of single dental implants: A 3-year prospective study. J Prosthet Dent.2019;1-6.

21. Zix, J., Hug, S., Kessler-Liechti, G. \& Mericske-Stern, R. Measurement of dental implant stability by resonance frequency analysis and damping capacity assessment: Com- parison of both techniques in a clinical trial. Int $\mathrm{J}$ Oral Maxillofac Implants 2008; 23: 525-30.

22. Al-Jetaily S, Al-Dosari AA. Assessment of Osstell ${ }^{\mathrm{TM}}$ and Periotest ${ }^{\circledR}$ systems in measuring dental implant stability (in vitro study). Saudi Dent J. 2011; 23(1):17-21.

23. Buyukguclu G, Ozkurt-kayahan Z, Kazazoglu E. Reliability of the Osstell Implant Stability Quotient and Penguin Resonance Frequency Analysis to evaluate implant stability. Dent Oral Health.2018.

24. Bilhan H, Cilingir A, Bural C, Bilmenoglu C, Sakar O, Geckili O. The Evaluation of the Reliability of Periotest for Implant Stability Measurements: An In Vitro Study. J Oral Implantol. 2015; 41(4):90-5.

25. Won-Suk Oh, Susan Almusa, Sheldon Winkler, Harold F. Morris. An in-vitro assessment of the reproducibility of Periotest value and implant stability quotient. J dent and oral health. Volume3. Issue 2. 2017; 061-04. 